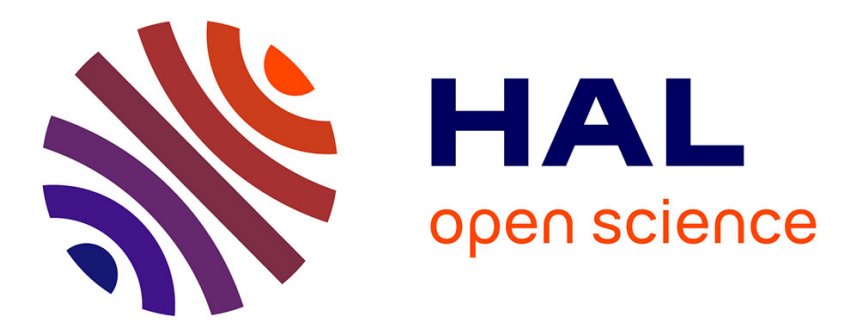

\title{
Quasi-automated reconstruction of the femur from bi-planar X-rays
}

François Girinon, Laurent Gajny, Shahin Ebrahimi, Louis Dagneaux, Philippe Rouch, Wafa Skalli

\section{- To cite this version:}

François Girinon, Laurent Gajny, Shahin Ebrahimi, Louis Dagneaux, Philippe Rouch, et al.. Quasi-automated reconstruction of the femur from bi-planar X-rays. Computer Methods in Biomechanics and Biomedical Engineering: Imaging \& Visualization, 2020, 8 (5), pp.529-537. 10.1080/21681163.2020.1725915 . hal-03131407

\section{HAL Id: hal-03131407 \\ https://hal.science/hal-03131407}

Submitted on 4 Feb 2021

HAL is a multi-disciplinary open access archive for the deposit and dissemination of scientific research documents, whether they are published or not. The documents may come from teaching and research institutions in France or abroad, or from public or private research centers.
L'archive ouverte pluridisciplinaire HAL, est destinée au dépôt et à la diffusion de documents scientifiques de niveau recherche, publiés ou non, émanant des établissements d'enseignement et de recherche français ou étrangers, des laboratoires publics ou privés. 


\title{
Quasi-automated reconstruction of the femur from bi-planar X-rays
}

\author{
François Girinon ${ }^{a}$, Laurent Gajny ${ }^{a}$, Shahin Ebrahimia ${ }^{a}$ Louis Dagneaux ${ }^{\mathrm{b}}$, Philippe Rouch ${ }^{\mathrm{a}}$ and Wafa Skallia

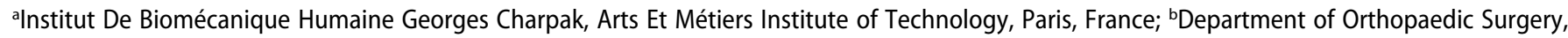 \\ CHU Montpellier, Montpellier, France
}

\begin{abstract}
$3 \mathrm{D}$ reconstruction from low-dose Bi-Planar X-Rays (BPXR) is a rising practice in clinical routine. However, this process is time consuming and highly depends on the user. This study aims to partially automate the process for the femur, thus decreasing reconstruction time and increasing robustness. As a training set, 50 femurs are segmented from CT scans together with 120 BPXR reconstructions. From this dataset, an initial solution for the bony contours is defined through Gaussian Process Regression (GPR), using eight digitized landmarks. This initial solution is projected on both x-rays and automatically adjusted using an adapted Minimal Path Algorithm (MPA). To evaluate this method, CT-scans were acquired from 20 cadaveric femurs. For each sample, the CTbased reconstruction is compared to the one automatically generated from the digitally reconstructed radiographs. Euclidean distances between femur reconstructions and the segmented CT data are on average $1.0 \mathrm{~mm}$ with a Root Mean Square Error (RMSE) of $0.8 \mathrm{~mm}$. Femoral torsion errors are assessed: the bias is lower than $0.1^{\circ}$ with a $95 \%$ confidence interval of $4.8^{\circ}$. The proposed method substantially improves 3D reconstructions from BPXR, as it enables a fast and reliable reconstruction, without the need for manual adjustments, which is essential in clinical routine.
\end{abstract}

\section{KEYWORDS}

Biomechanics; biomedical Imaging; computer Methods; femur; bi-planar X-rays; 3d reconstruction

\section{Introduction}

Three-dimensional reconstruction of the skeleton from biplanar X-rays (BPXR) is a rising practice in clinical routine. Compared to standard imaging techniques such as Computed Tomography (CT), this imaging modality enables a faster 3D reconstruction of the skeleton in the upright/ standing position. The reconstruction of the femur, based on bi-planar X-rays, is one of the most frequently discussed topics in the literature. For example (Youn et al. 2017) applied an iterative approach with uncalibrated X-rays yet. However, the patient is required to adopt a posture, which does not comply with the clinical standard. In a similar way to (Baka et al. 2011), the authors used canny edge detection for the segmentation process, which is known to be sensitive to noisy data. Furthermore, methods based on a database such as Active Shape Models (ASM) (Boussaid et al. 2011), Statistical Shape Models (SSM) (Dong et al. 2007; Baka et al. 2011; Cerveri et al. 2017; Zheng et al. 2018) or Statistical Shape and Intensity Models (Sadowsky et al. 2007; Väänänen et al. 2015; Klima et al. 2016) are widely considered, but do not allow to model pathological cases. On the other hand, algorithms based on free-form deformation (Laporte et al. 2003; Galibarov et al. 2010; Chaibi et al. 2012; Quijano et al. 2013; Karade and Ravi 2015) although less accurate for asymptomatic and nonsevere symptomatic cases, as less a priori knowledge is used, perform better for symptomatic patients. The method, used by (Quijano et al. 2013) is now applied on a routine basis in the clinical environment and uses the EOS system
(EOS Imaging, Paris, France), a calibrated system for the acquisition of low-dose BPXR. The system has been proven useful for pre-operative surgical planning, especially for patient monitoring through clinical parameters. The method considers a two-stage fast reconstruction process $(5 \mathrm{~min}$ for both lower limbs). First, the operator selects landmarks on both images. Based on these landmarks the geometrical parameters characterising the femur are inferred using partial least squares regression. An additional moving least squares (MLS) deformation is performed to obtain the initial solution. In the second stage, the operator adjusts the projected contours of the initial model with respect to the image contours. This is realised using handles to locally drive the deformation process through MLS. This method has been validated at the level of classical clinical parameters (mechanical femoral angle, hip-knee-shaft angle, cervico-diaphyseal angle, femoral torsion and length). However, to achieve a realistic representation of the bony surfaces, sufficient training is required. Note that fine manual adjustments are very time-consuming. To improve robustness and to speed up the process, automatising the 3D reconstruction process is necessary. The aim of this study is to introduce a novel approach to quasi-automatically reconstruct the femur from BPXR, thus removing the need for fine manual refinement.

To do so, the challenging task of automatically detecting the bone counters had to be resolved. Indeed, radiographs are noisy and bone structures overlay at some regions. The Minimal Path Algorithm (MPA) introduced by (Vincent 1998) 
was successfully applied to the femoral head (Ouertani et al. 2015). Also, Gaussian Process Regression (GPR) was recently proposed for shape models (Lüthi et al. 2016) and can be considered as a generalisation of (Laporte et al. 2003). However, it still might fail in case of multiple contours superimposition. Therefore, the proposed method combines an adapted MPA and GPR.

\section{Material and methods}

\subsection{Gaussian process regression}

GPR is a way to predict a posterior shape model composed of $n$ 3D anatomical landmarks out of which $m \leq n$ are known. Let $S_{i}, i=1, \ldots, N$ be $N$ 3D shapes, $S_{R}$ a reference shape and $\Omega$ a domain such that $S_{R} \subseteq \Omega$. Let us also consider deformation fields $\left\{u_{1}, \ldots, u_{N}\right\}, u_{i}: \Omega \rightarrow \mathbb{R}^{3}$ where $u_{i}(x)$ is the deformation field mapping the point $x \in S_{R}$ to the corresponding landmark $u_{i}(x)$ on $S_{i}$. We can, therefore, define a Gaussian process $\mathcal{G P}(\mu, k)$ which characterises deformations through the following mean function $\mu(x)$ and the covariance function $k(x)$ :

$$
\begin{aligned}
\mu(x) & =\frac{1}{N} \sum_{i=1}^{N} u_{i}(x) \text { and } k(x, y) \\
& =\frac{1}{N-1} \sum_{i=1}^{N}\left(u_{i}(x)-\mu(x)\right)\left(u_{i}(y)-\mu(y)\right)^{T}
\end{aligned}
$$

Assuming that for $m$ points, with location $P_{R}=\left\{p_{R}^{1}, \ldots, p_{R}^{m}\right\}, p_{R}^{l} \in$ $\mathbb{R}^{3} \mid I=1, \ldots m$ on the reference surface, the corresponding location on the target surface is defined as $P_{T}=\left\{p_{T}^{1}, \ldots, p_{T}^{m}\right\}, p_{T}^{s} \in$ $\mathbb{R}^{3} \mid s=1, \ldots m$. Hence, the deformation for each of these matching points can be calculated as follows:

$$
\begin{aligned}
L & =\left\{\left(p_{R}^{1}, p_{T}^{1}-p_{R}^{1}\right), \ldots,\left(p_{R}^{m}, p_{T}^{m}-p_{R}^{m}\right)\right\}: \\
& =\left\{\left(p_{R}^{1}, \hat{u}^{1}\right), \ldots,\left(p_{R}^{m}, \hat{u}^{m}\right)\right\}
\end{aligned}
$$

where $\hat{u}_{l}, I=1$ are the deformations subject to a Gaussian noise $\varepsilon \sim \mathcal{N}\left(0, \sigma^{2} I_{3}\right)$, and $l_{3} \in \mathbb{R}^{3 \times 3}$ is the identity matrix of order 3 . We can now use these prior deformations to obtain from $\mathcal{G P}(\mu, k)$ a new Gaussian process known as a posterior model $\mathcal{G P}(\bar{\mu}, \bar{k})$. The posterior mean $\bar{\mu}$ and the posterior covariance $\bar{k}$ can be computed in closed form:

$$
\begin{aligned}
& \bar{\mu}(x)=\mu(x)+K_{X}(x)^{T}\left(K_{X X}+\sigma^{2} l_{3}\right)^{-1} \hat{U} \\
& \bar{k}\left(x, x^{\prime}\right)=k\left(x, x^{\prime}\right)-K_{X}(x)^{T}\left(K_{X X}+\sigma^{2} I_{3}\right)^{-1} K_{X}\left(x^{\prime}\right)
\end{aligned}
$$

with $K_{X}(x)=\left(k\left(x, p_{R}^{l}\right)\right)_{l=1}^{m} \in \mathbb{R}^{3 n \times 3}, K_{X X}=\left(k\left(p_{T}^{s}, p_{R}^{l}\right)\right)_{s, l=1}^{m} \in$ $\mathbb{R}^{3 m \times 3 m}, \hat{U}=\left(\hat{u}^{1}-\mu(x), \ldots, \hat{u}^{m}-\mu(x)\right)^{T} \in \mathbb{R}^{3 n}$.

\subsection{Minimal path algorithm}

MPA (Vincent 1998; Ouertani et al. 2015) has been proven to be a robust way to detect linear features in grey images using an initial solution. Starting from an initial contour denoted $C_{0}$, the method looks for a similar shape in a userdefined searching zone. The initial contour is first uniformly subsampled, resulting in $n_{s} \in \mathbb{N}$ points $s_{i}, i=1, \ldots, n_{s}$. Then, for each $s_{i}, n_{e}\left(n_{e} \in \mathbb{N}\right)$, the points $q_{i j}, j=1, \ldots, n_{e}$, are sampled along the normal, on both sides of the contour, defining a ribbon (see Figure 1). Intensity values at each $q_{i j}$ are obtained by linear interpolation of the pixel intensity table and an image of size $n_{s} \times n_{e}$ can be built. To look for the contour, the gradient map is computed and an oriented graph is built, connecting all adjacent pixels and penalising the pixels of diagonal. This yields a cost map. Finally, the searched contour is the path that minimises the sum values of the nodes traversed in this graph.

\subsection{Subjects and database}

A first Gaussian Process denoted $G P_{1}$ is built from 120 femurs manually reconstructed from bi-planar X-rays (Nerot et al. 2017). They are aligned on their barycentre, encoding this way the rigid rotation prior. Additionally, 50 segmented femurs are collected from the Virtual Skeleton Database (Kistler et al. 2013). These femurs are used to build a second Gaussian Process denoted $G P_{2}$. They are registered with the same method than Schlager and Rüdell (2017) and aligned through the Generalised Procrustes Analysis.

The second part of our database is dedicated to the validation process and is completely independent from the previous one. Twenty femurs were segmented from cadaveric CT-scans $(0.75 \mathrm{~mm}$ thickness) using MITK-GEM (Pauchard et al. 2016) (16 intact and 4 arthritic ones). Additionally, 24 (mean age 27 year, 12 subjects, 6 males, 6 females) healthy and 16 (mean age 67 year, 10 subjects, 6 males, 4 females) pathological (osteoarthritis) femurs are also reconstructed from bi-planar X-rays $(0.186 \mathrm{~mm}$ resolution) using the method described in (Quijano et al. 2013). Another set of 30 (mean age 55 years, 5 asymptomatic patients, 10 arthritic ones) bi-planar X-rays was considered for a reproducibility study. Note that all bi-planar X-rays in the different databases were acquired using the low-dose EOS-calibrated system (EOS imaging, Paris, France).

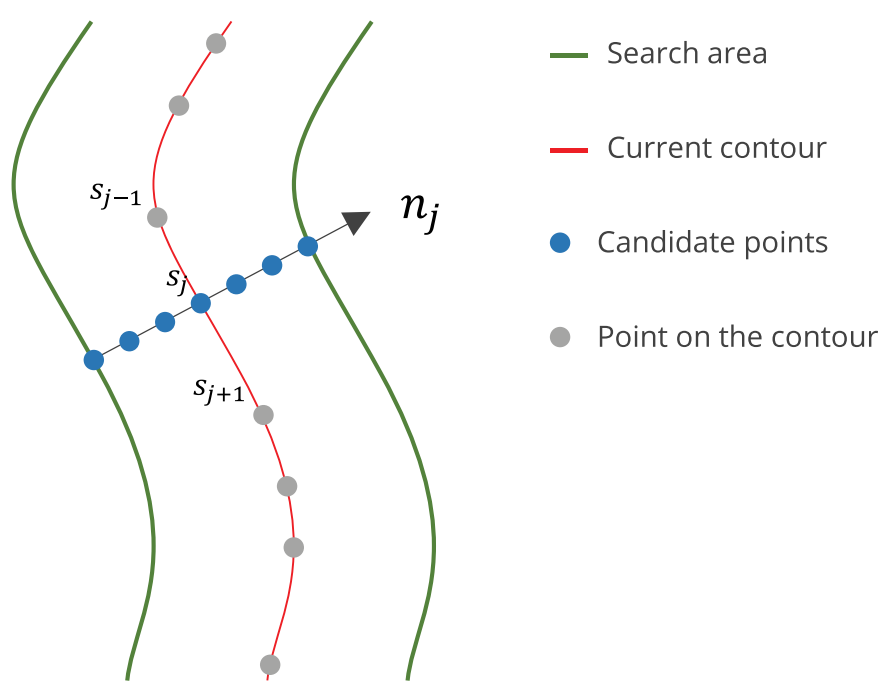

Figure 1. Sampling process of the ribbon. 


\subsection{Initial solution}

Eight radiological landmarks (Figure 2) were digitised on each $X$-rays: one Stereo-Corresponding (SCP) landmark (one sphere for the femoral head), four Non-Stereo-Corresponding Points (NSCP) on the frontal view (greater and lesser trochanter and the mediolateral points of the condyles), two on the sagittal view (the two posterior condyle points). Note that there is no need to identify which posterior condyle point is medial or lateral.

At this stage, the SCP is used through the Gaussian process regression using $G P_{1}$ (ie. computing the posterior mean according to (3)). One of the landmarks is randomly assigned to the medial posterior points, the other one as the lateral posterior landmark. Then, two successive GPR are applied, using first the posterior points, then the lateral one. Contrary to the previous point, they are NSCP, only the line they belong to is known. Therefore, to get an approximate $3 \mathrm{D}$ location of the vertex, the corresponding one on the current femur shape is projected onto this line. The two possible configurations for the assignment of medial and lateral condyles are tested and the one with the highest probability shape score regarding the Gaussian process is kept.

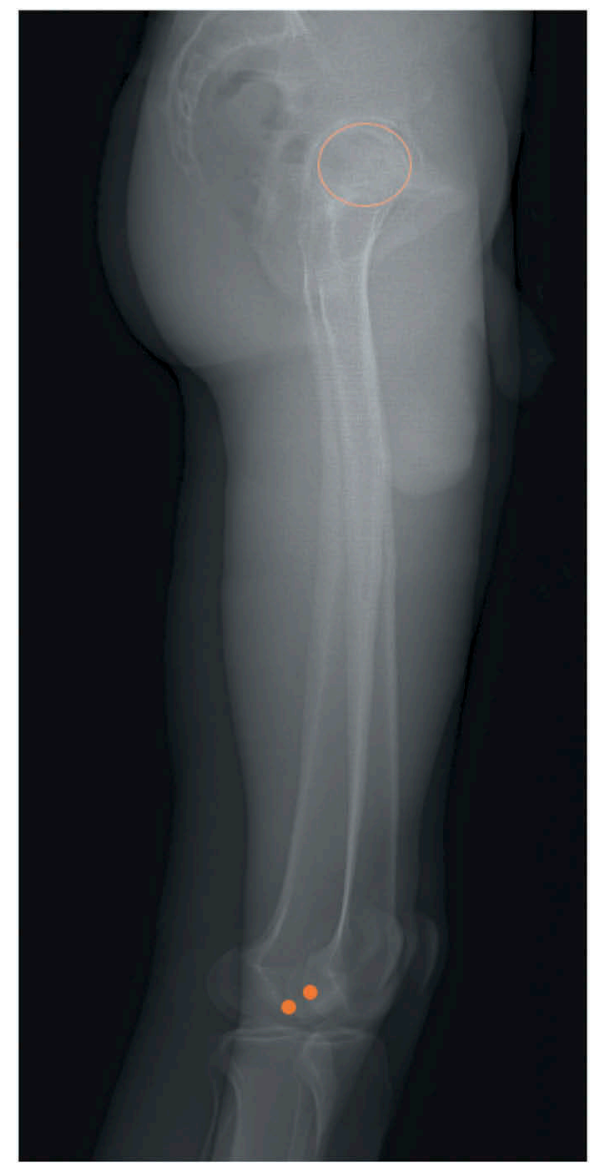

(a) Sagittal view

\section{5. $3 D$ reconstruction algorithm}

Once the initial solution is obtained, it is projected onto the radiographs and femur contours can be computed. In the contour extraction process of the mesh, two kinds of contours are considered, the external ones which basically correspond to the silhouette and the internal ones which are generated from local bumps. Internal contours are computed in a similar way to (Dong et al. 2007). The outer contours of the projected mesh are obtained first projecting all triangle faces, then iteratively merging them using Vatti's algorithm (Vatti 1992). This process ensures a clean silhouette extraction and enables identification of the vertices belonging to this one.

A recursive median filter and an adaptive histogram equaliser filter are first applied on the region of interest. Then, a modified MPA is applied, followed by a deformation step restricted to some regions $R_{i}$ depending on the iteration $i$. While the maximum displacement condition is not reached or $i<20$, the process iterates again (Figure 3 , Algorithm 1).

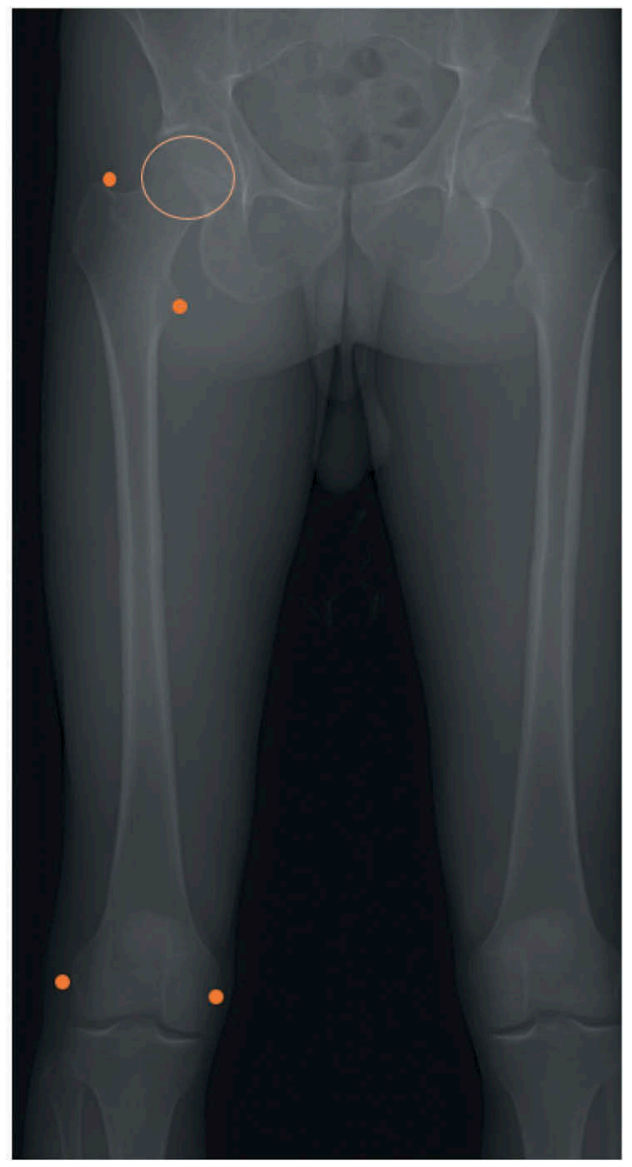

(b) Frontal view

Figure 2. Initial digitisation of the femur from bi-planar X-rays. (a) From proximal to distal, the annotated landmarks are the femoral head and posterior condyle points. (b) From proximal to distal, the annotated landmarks are the femoral head, the greater trochanter, the lesser trochanter, medial and lateral extreme points of the condyles. 


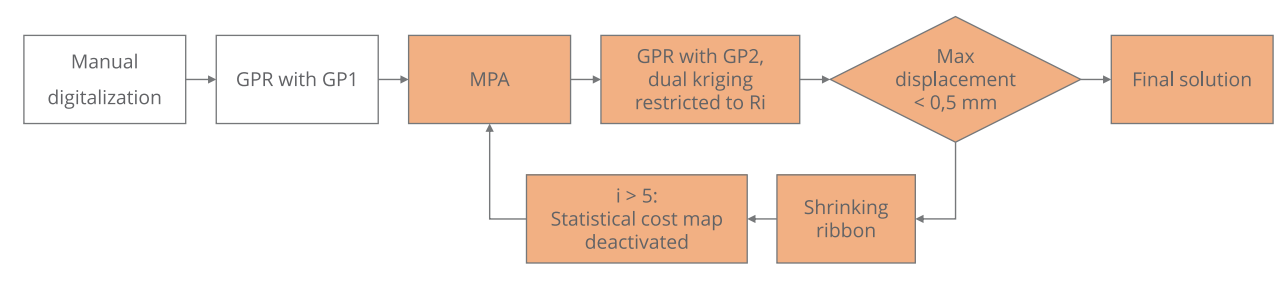

Figure 3. Whole pipeline of the reconstruction process.

Algorithm 1 Pseudo code of the reconstruction process

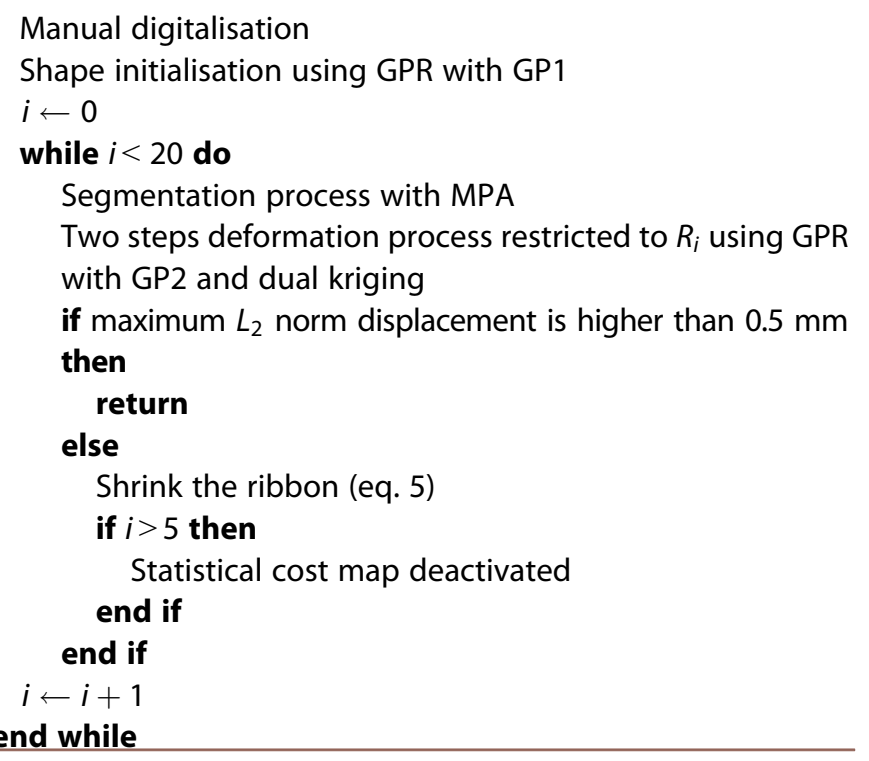

Modifications in the minimal path algorithm consist of the introduction of two additional cost maps to increase robustness to noise and superimposition of structures. A cost map is introduced to constrain the path to go through digitised landmarks. To do so, we first define the uncertainty range $\sigma$ related to the operator's expected accuracy for each point (Schlatterer et al. 2009). All weights of the cost map around the selected landmarks in a $\sigma$ range are set as infinite values. Then, the cost map in the neighbourhood of these landmarks is also weighted again with a Gaussian filtering operation with the same $\sigma$ parameter. This aims to smoothly drive the path near the operator selection.

The last cost map is a statistical one. To each candidate point on $n_{j}$, a constraint line can be associated. And, to each of this constraint line a 3D point can be calculated as the projection of the $3 \mathrm{D}$ shape onto this line. Then, considering the posterior $G P_{1}$ with a mean $\bar{\mu}$ and a kernel $\bar{\Sigma}$, for two neighbour candidates $\left(c_{1}, c_{2}\right) \in \mathbb{R}^{3}$ along $n_{j}$, similar to (Huang et al. 2015), the paired statistical energy is defined as:

$$
E_{\text {stat }}\left(c_{1}, c_{2}\right)=\frac{1}{2} \sum_{i=1}^{2}\left(\exp \left(-\frac{1}{2}\left(c_{i}-\bar{\mu}\right) \bar{\Sigma}^{-1}\left(c_{i}-\bar{\mu}\right)^{T}\right)\right)
$$

This energy is particularly efficient to discriminate outliers. For example, on the proximal part, when diaphyses are close from each other, and parallel enough, the statistical cost function ensures the right contour of the right diaphysis is detected.
Finally, the directed graph can be solved using dynamic programming as proposed in (Ouertani et al. 2015) (Figure 4).

At this stage, the MPA finds a contour which follows the gradient edges. Thanks to the sampling process, paired points between the current contour and the one which is detected are automatically set. To compute a plausible new 3D location of each vertex belonging to the contour, the associated one to the current 3D shape is projected on the constraint line coming from the image paired point. The vertices which belong to the contour are used in a GPR with $G P_{2}$. It is, therefore, necessary to also rigidly align the new matched vertices coordinates with the mean shape. For that reason, General Procrustes Analysis (GPA) is realised between the matched vertices and the corresponding mean ones of the GP. To model uncertainty of the new locations of the concerned vertices, anisotropic Gaussian noise is introduced with a variance of $20 \mathrm{~mm}^{2}$ in the direction of the constrained line, $2 \mathrm{~mm}^{2}$ otherwise. A second deformation stage is achieved; this time, a dual kriging (Trochu 1993). This enables to capture finer details and optimise the global position of the bone. Finally, the obtained shape is projected and the same last steps are applied again.

Because particular matched regions are not reliable at first sight, they are deactivated during the first iterations. For example, the lesser trochanter and the anterior distal part near the patella are activated after five iterations. Meanwhile, these regions are driven by the GPR and therefore get closer and closer from the target shape.

Simultaneously to this process, the ribbon is shrunk, discarding outliers in noisy areas. Considering $r_{w_{0}}$ as the initial ribbon width, $s_{f}$ the shrinking factor and $i$ the number of iterations,

$$
r_{w}=r_{w_{0}} s_{f}^{i}
$$

$r_{w_{0}}$ and $s_{f}$ are arbitrary defined through a trial-and-error methodology Table 1. For instance, $r_{w_{0}}$ is set to $120 \mathrm{px}$ and $s_{f}$ to 0.9 for the frontal silhouette.

\subsection{Evaluation}

The $3 \mathrm{D}$ reconstruction is first evaluated in terms of shape and femoral torsion accuracy. For each cadaveric CT-scan, a 3D mask has been drawn to remove one lower limb, as the two femurs are each time strictly aligned. From these masked 3D volumes, digitally reconstructed radiographs (DRR) are created to simulate bi-planar radiographs with the same calibrated radiological environment of the EOS.

The 3D reconstruction of the femur from DRRs is then compared to a segmented object considered as the gold standard. As 


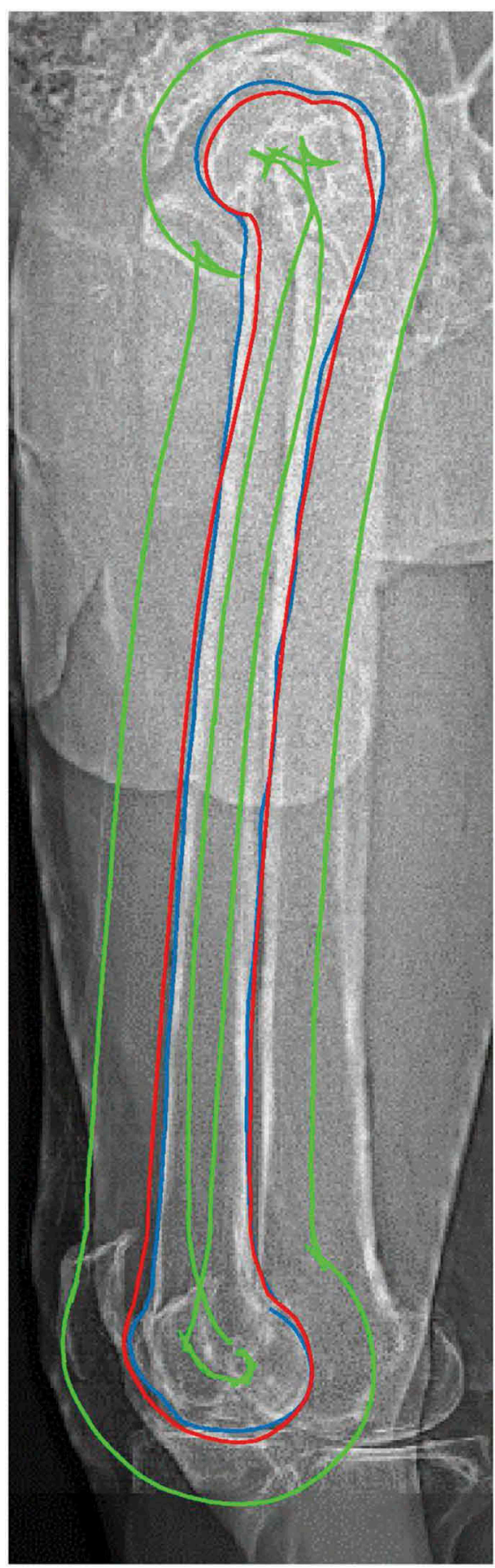

(a) Sagittal view

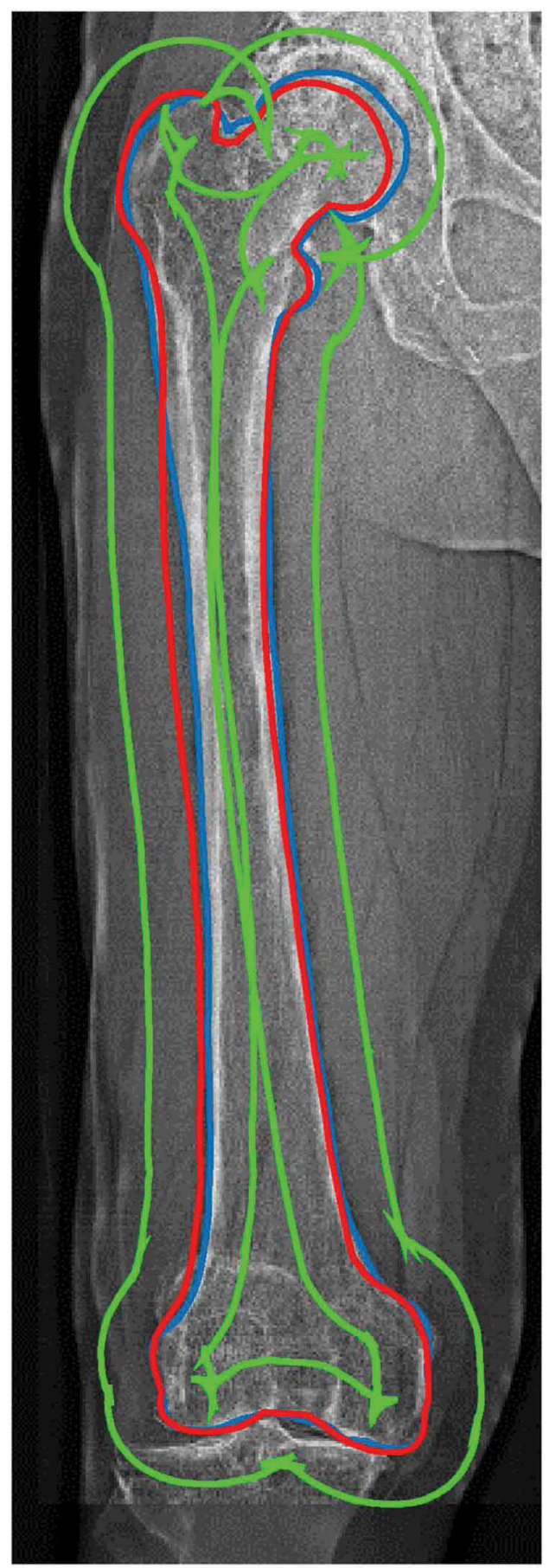

(b) Frontal view

Figure 4. Result of the MPA using the projected contours of the initial solution - red, contour of the initial solution - green, search area - blue, new detected contour.

Table 1. Ribbon parameters defined through trial-and-error methodology for each contour region.

\begin{tabular}{lcccc}
\hline Contour region & Frontal view & Sagittal view & $r_{w_{0}}(\mathrm{px})$ & $s_{f}$ \\
\hline Femur silhouette & & $\mathrm{x}$ & 120 & 0.9 \\
Femur silhouette & $\mathrm{x}$ & & 100 & 0.9 \\
Greater trochanter & & $\mathrm{x}$ & 40 & 1.0 \\
Trochlea & & $\mathrm{x}$ & 30 & 1.0 \\
Condyles & & $\mathrm{x}$ & 40 & 0.95 \\
\hline
\end{tabular}

the DRR is generated in the EOS environment, point-to-surface distances can be computed directly. All the reconstructed femurs have the same topological mesh since they are generated from the GP. Therefore, a distance map is calculated projecting the 2372 vertices onto the target segmentation. At each of these vertices, mean and the Root Mean Square Error (RMSE) are calculated. The global mean error is also estimated and twice the RMSE is 
considered as the shape uncertainty. Femoral torsion, considered as the major clinical parameter is automatically extracted from both shapes, our 3D reconstruction and the gold standard.

The semi-automated 3D reconstruction is also evaluated on real bi-planar X-rays. From the database of 40 patients, this method and Quijano et al. 2013 method have been both applied and compared in terms of femoral torsion and time. A Bland-Altman plot (Altman and Bland 1983) is also used to compare the two methods.

Finally, the 15 bi-planar X-rays aim to assess the reproducibility of our method. Each reconstruction of the femur was performed once by three different operators (a beginner, an intermediate and an expert). The entire protocol follows the ISO 5725-2 standard.

\section{Results}

\subsection{Comparison to the CT-scan}

The point-to-surface distance between the 3D quasi-automated reconstruction of the femur (Figure 5), and the 3D reference shows a global mean value of $1.0 \mathrm{~mm}$ and 2 RMSE $1.6 \mathrm{~mm}$. The higher errors appear on the interior part of the greater trochanter. The femoral torsion error is presented through the BlandAltman plot (Figure 6). The bias of the femoral torsion error is reported as $0.1^{\circ}$ (2.2 for (Quijano et al. 2013)) and the 2 standard deviation (SD) as $4.7^{\circ}$ (not defined in (Quijano et al. 2013)).

\subsection{Comparison to the previous method}

The bias of the computed femoral torsion parameter obtained with our method as compared to the one obtained from (Quijano et al. 2013) is reported as $-1.1^{\circ}$ with a 2 SD of $5.5^{\circ}$. As previously, the Bland-Altman plot provides a more detailed overview (Figure 7). Note the 2 SD reproducibility error in (Quijano et al. 2013) study is estimated as $3.8^{\circ}$. With respect to the reconstruction times previously reported (for both lower limbs) as 3-4.6 min, we speed up the manual landmark annotation (Figure 3) process (2.5-3.5 min). These lower and higher time values are calculated on this same database.

\subsection{Reproducibility study}

The reproducibility study involving 3 operators aims to compute (Table 2) the standard deviations of reproducibility according to the ISO 5725-2 standard.

\section{Discussion}

The aim of this study was to introduce a quasi-automated reconstruction method for the femur using bi-planar X-rays.

\subsection{D reconstruction method}

The reconstruction method of (Quijano et al. 2013) required two steps: initial solution and contour adjustment. The reconstruction pipeline proposed here only needs $2 \mathrm{D}$ digitisation; adjustment is now fully automated reducing operator time. Regarding the initial solution, it is less operator-dependent since medio-lateral condyles do not have to be distinguished
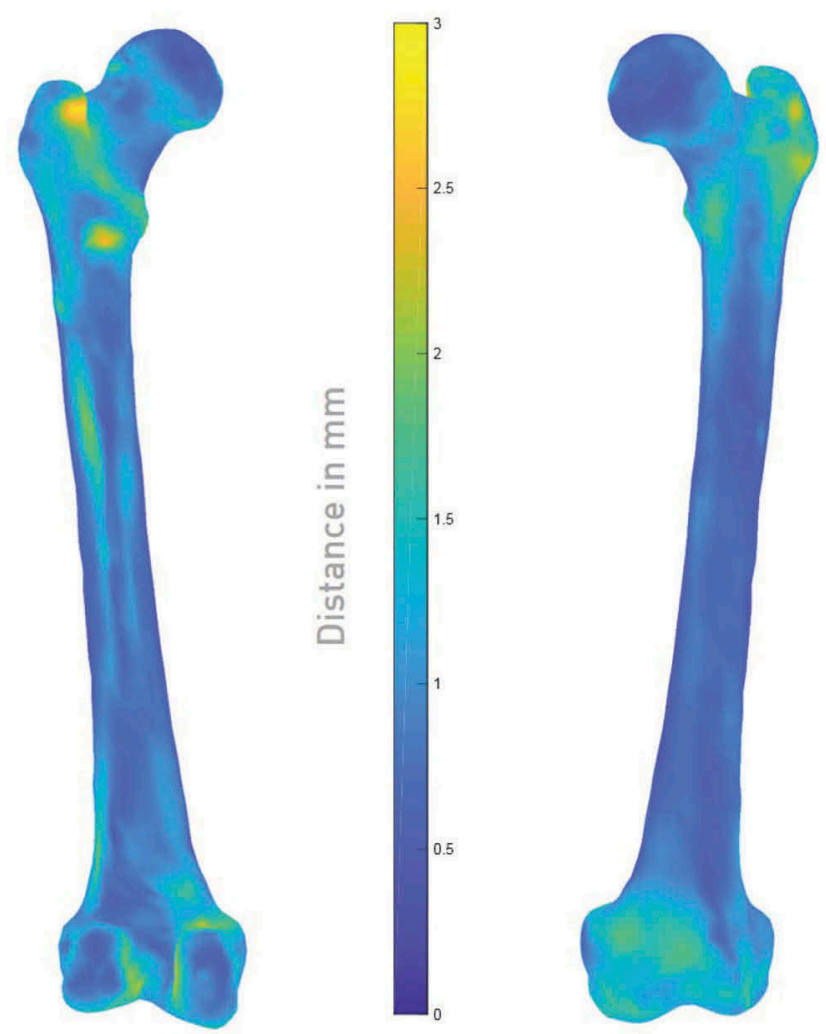

(a) distance map of the mean errors

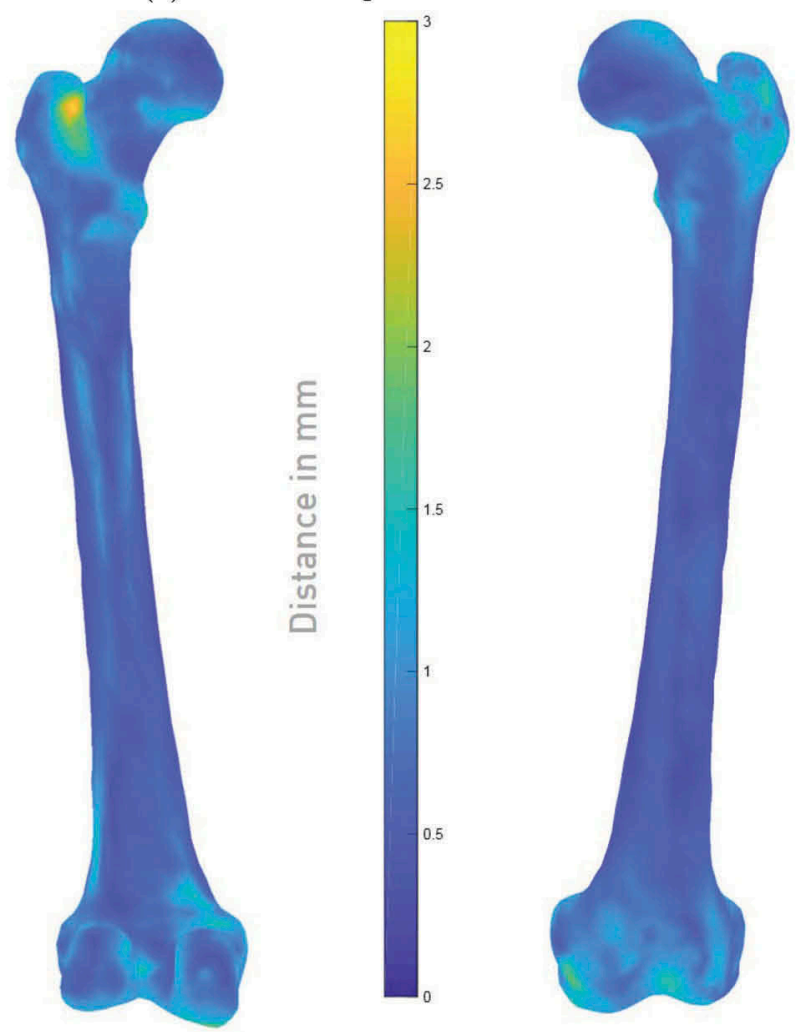

(b) distance map of the RMSE

Figure 5. Points-to-surface metrics comparing the proposed method to the gold standard.

anymore. Meanwhile, the number of radiological landmarks have been lowered ( $40 \mathrm{~s}$ to select them against a minute for the previous approach). 


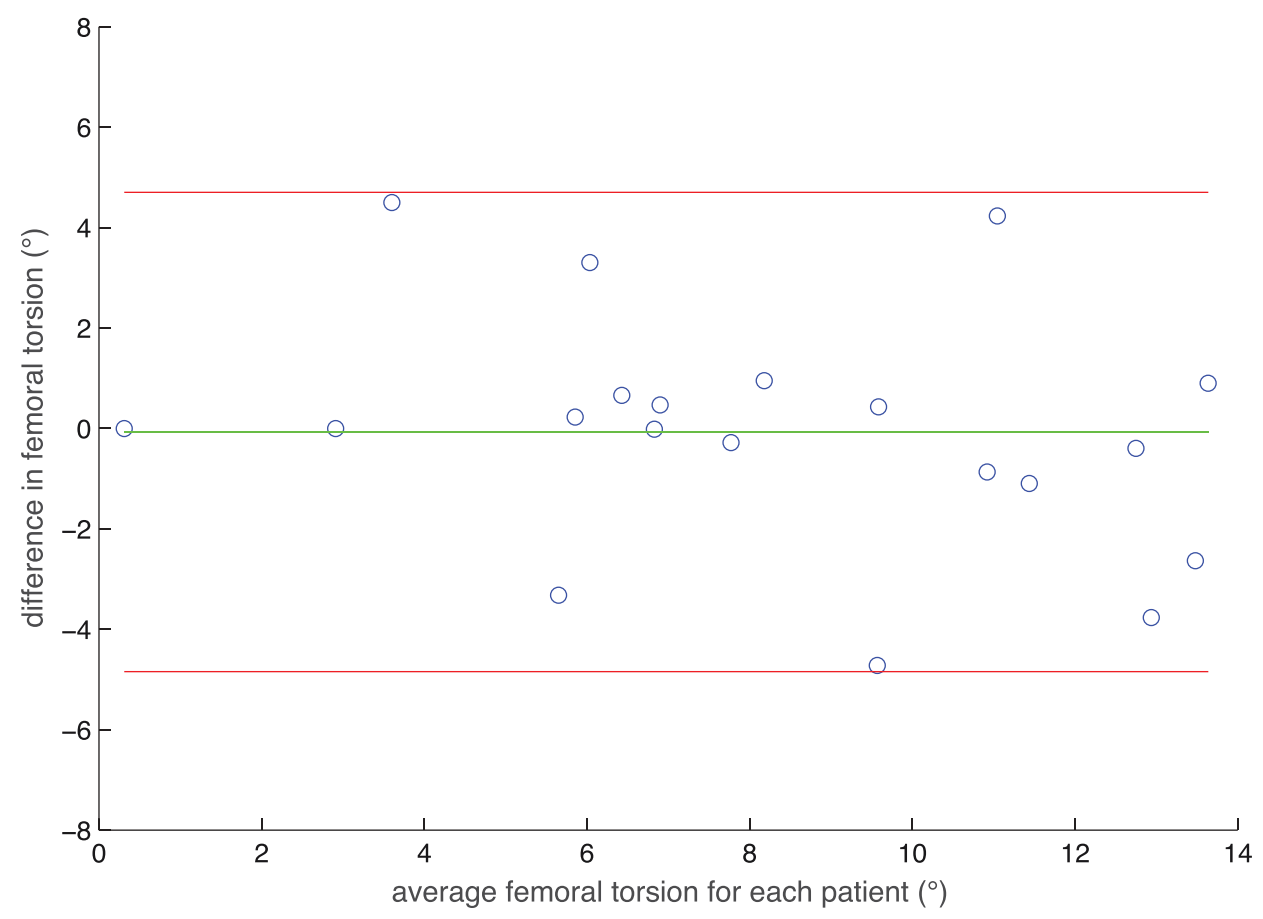

Figure 6. Bland-Altman plot comparing computed femoral torsion from the proposed method to the gold standard - bias in green - 2SD in red.

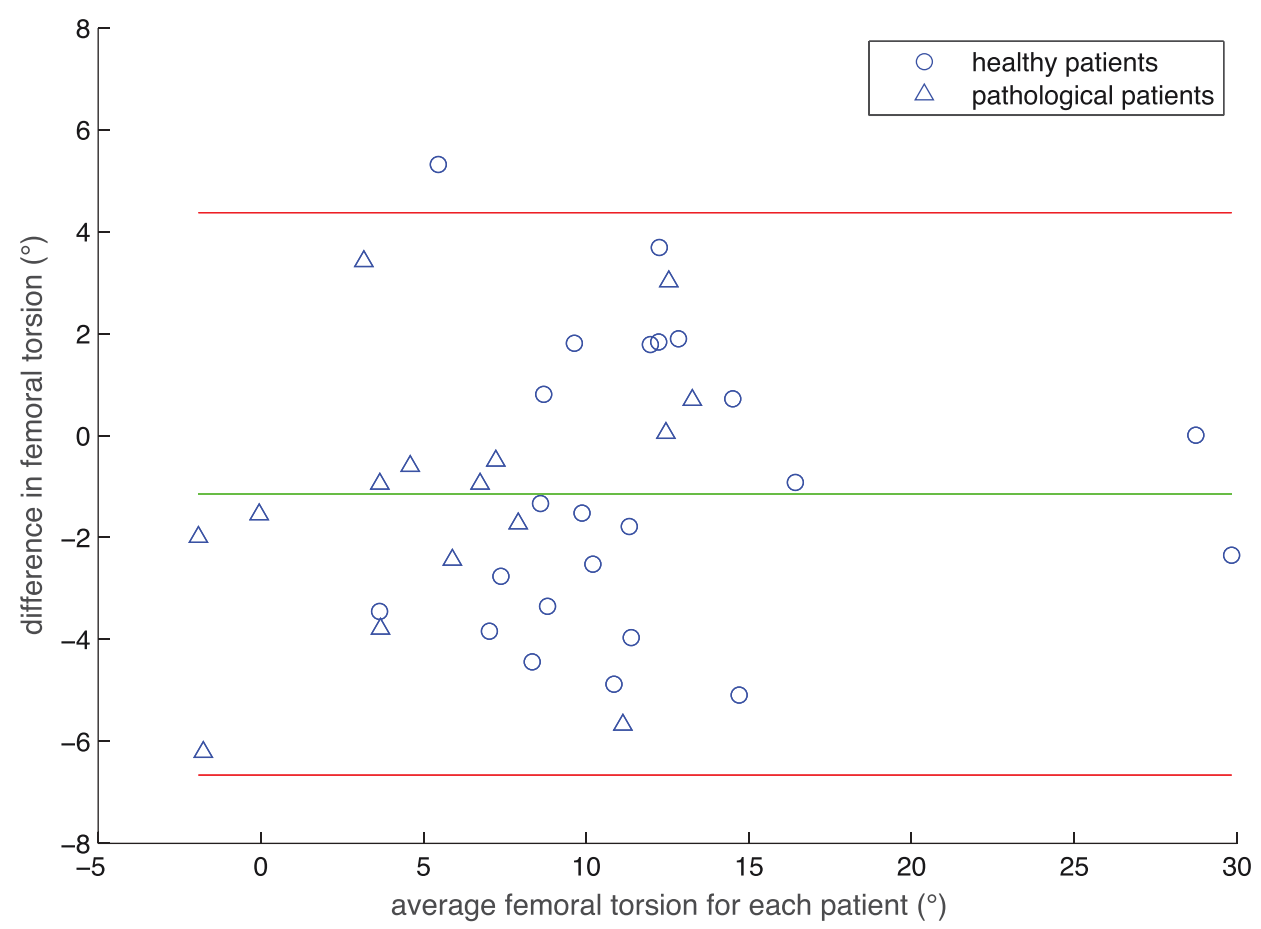

Figure 7. Bland-Altman plot comparing computed femoral torsion from the proposed method to (Quijano et al. 2013) - bias in green - 2SD in red.

Moreover, automated segmentation has been achieved combining a minimal path algorithm with different prior knowledge, GPR and dual kriging. This overcomes the issue of containment (Lüthi et al. 2016) to the database on which SSM-based methods are usually subject to.

\subsection{Metrics accuracy}

Compared to the literature the proposed method is the best trade-off between simplicity and robustness. Few studies dealt with the entire femur. Among them, in term of points-tosurface distances, they, respectively, obtained for the mean 
Table 2. Two SD of a series of femoral clinical parameters derived from a database of healthy and pathological patients reconstructed from bi-planar X-rays using our method. Comparison of our proposed method with the previous one (Quijano et al. 2013).

\begin{tabular}{lcccc}
\hline Clinical parameters & \multicolumn{2}{c}{$\begin{array}{c}\text { Asymptomatic } \\
\text { previous/current }\end{array}$} & \multicolumn{2}{c}{$\begin{array}{c}\text { Pathological } \\
\text { previous/current }\end{array}$} \\
\hline Mechanical femoral angle $\left(^{\circ}\right)$ & 1.3 & 1.2 & 1.4 & 1.3 \\
Hip-knee-shaft angle $\left(^{\circ}\right)$ & 0.8 & 0.4 & 0.5 & 0.5 \\
Cervico-diaphyseal angle $\left(^{\circ}\right)$ & 3.8 & 2.4 & 4.5 & 3.0 \\
Femoral torsion $\left({ }^{\circ}\right)$ & 3.0 & 1.0 & 3.7 & 1.8 \\
Femoral length $(\mathrm{mm})$ & 1.8 & 0.9 & 1.7 & 1.3 \\
\hline
\end{tabular}

and 2 RMSE: (Klima et al. 2016) $(1.0 \mathrm{~mm}, 1.35 \mathrm{~mm})$, (Chaibi et al. 2012; Quijano et al. 2013) (1.0 mm, $2.4 \mathrm{~mm})$, (Zheng 2010) $(1.1 \mathrm{~mm}, 2.8 \mathrm{~mm})$ and our method $(1.0 \mathrm{~mm}, 1.6 \mathrm{~mm})$.

Regarding the proposed $3 \mathrm{D}$ reconstruction, maximum errors appeared on the inner part of great trochanter, but this will not impact clinical parameters. Besides, femoral torsions measured on cadaveric subjects were close from the values obtained with the previous method. We also successfully applied our method in real conditions and for pathological cases which has rarely been reported in the literature (Chaibi et al. 2012; Quijano et al. 2013). We then compared the femoral torsions to those obtained in (Quijano et al. 2013) and did not notice any real differences between pathological and healthy patients. However, the errors were seen little higher compared to cadaveric subjects.

The reproducibility study shows for every clinical parameters a systematic precision gain compared to previous works. It is also important to notice here that only one operator was an expert. This proved a short training $(30 \mathrm{~min}$ ) is sufficient to obtain results close to those of the expert.

\subsection{Limitations and future works}

As main limitation of this study, only the femur is considered and not the entire lower-limb. Furthermore, shape accuracy is only validated on DRR but we expect even better results in real clinical environment since bi-planar images have higher contrast and resolution. Another limitation of this approach and the previous ones is to consider femur shapes as a continuous representation. Therefore, discontinuous structures such as fractures, or unfused bones in children cannot be modelled with these methods. This may be solved if we were able to segment the different bone fragments and deform each separated part. Still, it would be necessary to assess our reconstruction process on children to verify such outliers with regard to the database can also be accurately reconstructed. Furthermore, small sharp features such as osteophytes cannot be accurately modelled since our minimal path algorithm tends to consider them as noise. Considering local abnormalities is part of future work. The whole process takes $3 \mathrm{~min}$ in a non optimised MATLAB version. The most time-consuming part lies in the projection of the contours and will have to be improved in future works.

To speed up the reconstruction process, it would also be interesting to compare the greedy dynamic programming used with a stochastic solver even though global convergence would be no longer insured. The new pipeline we design leads to a robust detection of the contours thanks to a shrinking ribbon mechanism and a region activation/deactivation strategy. Therefore, MPA is well suited for these tasks. However, further investigation will be led to compare different methods for segmentation purposes.

\section{Conclusion}

A quasi-automated method of 3D reconstruction of the femur from bi-planar X-rays has been introduced, reducing the process of manual annotations and deleting the fine manual adjustment phase. A new set of landmarks has been proposed. In particular, the operator is not asked anymore to perform the challenging and error-prone task of identifying medial and lateral condyles on the sagittal view. Forty seconds are needed to identify the landmarks against a minute for the method available in clinical routine. More benefits are obtained on the refinement stage which is now strictly automated ( 2 min by femur using the previous approach). The method has been proven to be accurate and reproducible in terms of shape and clinical parameters. It should be extended soon to the full lower limb and pelvis.

\section{Acknowledgments}

We would like to thank Maxim Van den Abbeele and Bhrigu Lakhar for their critical comments, which greatly improved the quality of this paper.

\section{Disclosure statement}

No potential conflict of interest was reported by the authors.

\section{Notes on contributors}

François Girinon received his master degree in Biomedical Enginering from Arts et ParisTech in 2015 and the PhD degree in Biomedical imaging from Arts et Métiers ParisTech in 2018. His general interests lie in geometric modeling and medical image analysis for the 3D reconstruction of the lower limbs.

Laurent Gajny is assistant professor in applied mathematics at Arts et Métiers ParisTech. His research interests are in numerical analysis and geometric modelling applied to the $3 \mathrm{D}$ reconstruction of the human body from medical images.

Shahin Ebrahimi received his master degree in Biomedical Enginering from Telecom ParisTech in 2014 and the PhD degree in Computer Science from Arts et Métiers ParisTech in 2017. His general interests lie in machine learning, pattern recognition and their application to computer vision and medical image analysis.

Senior consultant and Assistant Professor in Orthopedic surgery (Montpellier, France) for five years, Louis Dagneaux is currently working at the Mayo Clinic as visiting scientist. With the support of over 25 peerreviewed publications and 3 book chapters, he focuses his research and clinical practice on patellofemoral factors related to knee arthroplasty and arthroscopic foot and ankle surgery using mechanics of the lower-limb.

Professor Philippe Rouch is Paris Campus Director of the Arts et Métiers ParisTech. He has also been director of the Institut de Biomécanique humaine Georges Charpak in Art et Métiers ParisTech. He is particularly involved in musculoskeletal modeling with a strong interest in kinematic analysis.

Wafa Skalli is a professor in biomechanics at Arts et Métiers ParisTech. She is founder and scientific director of the Institut de Biomécanique Humaine Georges Charpak in Arts et Métiers ParisTech, and holder of the BiomecAM ParisTech chair on subject-specific musculoskeletal modelling. She is particularly involved in biomechanics and modelling of the spine, with a strong link to experimental and clinical approach. 


\section{References}

Altman DG, Bland JM. 1983. Measurement in medicine: the analysis of method comparison studies. The Statistician. 3:307.

Baka N, Kaptein BL, de Bruijne M, van Walsum T, Giphart JE, Niessen WJ, Lelieveldt BPF. 2011. 2d-3d shape reconstruction of the distal femur from stereo $\mathrm{x}$-ray imaging using statistical shape models. Med Image Anal. 6:840-850.

Boussaid H, Kadoury S, Kokkinos I, Lazennec JY, Zheng G, Paragios N. 2011. $3 \mathrm{D}$ model-based reconstruction of the proximal femur from low-dose Biplanar X-Ray Images. BMVC. 35:1-35.10.

Cerveri P, Sacco C, Olgiati G, Manzotti A, Baroni G. 2017. 2d/3d reconstruction of the distal femur using statistical shape models addressing personalized surgical instruments in knee arthroplasty: A feasibility analysis. Int J Med Robot. 13(4):e1823.

Chaibi Y, Cresson T, Aubert B, Hausselle J, Neyret P, Hauger O, de Guise JA, Skalli W. 2012. Fast 3d reconstruction of the lower limb using a parametric model and statistical inferences and clinical measurements calculation from biplanar x-rays. Comput Methods Biomech Biomed Engin. 5:457-466.

Dong X, Gonzlez Ballester M, Zheng G. 2007. Automatic extraction of femur contours from calibrated $x$-ray images using statistical information. J Multimed. 2(5):46-54.

Galibarov PE, Prendergast PJ, Lennon AB. 2010. A method to reconstruct patient-specific proximal femur surface models from planar pre-operative radiographs. Med Eng Phys. 10:1180-1188.

Huang J, Griffith JF, Wang D, Shi L. 2015. Graph-cut-based segmentation of proximal femur from computed tomography images with shape prior. J Med Biol Eng. 35(5):594-607.

Karade V, Ravi B. 2015. 3d femur model reconstruction from biplane $x$-ray images: a novel method based on laplacian surface deformation. Int J Comput Assist Radiol Surg. 4:473-485.

Kistler M, Bonaretti S, Pfahrer M, Niklaus R, Bchler P. 2013. The virtual skeleton database: an open access repository for biomedical research and collaboration. J Med Internet Res. 15(11):e245.

Klima O, Kleparnik P, Spanel M, Zemcik P. 2016. Intensity-based femoral atlas $2 \mathrm{~d} / 3 \mathrm{~d}$ registration using levenberg-marquardt optimisation. Proceed SPIE. 9788:97880F. id 97880F 12 pp.

Laporte S, Skalli W, de Guise J, Lavaste F, Mitton D. 2003. A biplanar reconstruction method based on $2 \mathrm{~d}$ and $3 \mathrm{~d}$ contours: application to the distal femur. Comput Methods Biomech Biomed Engin. 6(1):1-6.

Lüthi M, Jud C, Gerig T, Vetter T, Luthi M, Gerig T, Jud C, Vetter T. 2016. Gaussian process morphable models. IEEE Trans Pattern Anal Mach Intell. 40(April): 1-38.

Nerot A, Skalli W, Wang X. 2017. Estimation of spinal joint centers from external back profile and anatomical landmarks. Journal of Biomechanics. 70:96-101.
Ouertani F, Vazquez C, Cresson T, De Guise J 2015. Simultaneous extraction of two adjacent bony structures in $\mathrm{x}$-ray images: application to hip joint segmentation. Proceedings - International Conference on Image Processing, ICIP; 2015 December(January); Quebec City, Canada. p. 4555-4559.

Pauchard Y, Fitze T, Browarnik D, Eskandari A, Pauchard I, Enns-Bray W, PIsson H, Sigurdsson S, Ferguson SJ, Harris TB, et al. 2016. Interactive graph-cut segmentation for fast creation of finite element models from clinical ct data for hip fracture prediction. Comput Methods Biomech Biomed Engin. 19(16):1693-1703.

Quijano S, Serrurier A, Aubert B, Laporte S, Thoreux P, Skalli W. 2013. Threedimensional reconstruction of the lower limb from biplanar calibrated radiographs. Med Eng Phys. 12:1703-1712.

Sadowsky O, Chintalapani G, Taylor RH, van der Bom MJ, Pluim JPW, Homan R, Timmer J, Bartels LW 2007. Deformable 2d-3d registration of the pelvis with a limited field of view, using shape statistics. Lecture Notes in Computer Science (including subseries Lecture Notes in Artificial Intelligence and Lecture Notes in Bioinformatics), MICCAI 2007, 10th International Conference; Oct 29-Nov 2; Brisbane, Australia. (Pt 2): p. 65120B-65120B-9.

Schlager S, Rüdell A. 2017. Sexual dimorphism and population affinity in the human zygomatic structure-comparing surface to outline data. Anatomical Record. 300(1):226-237.

Schlatterer B, Suedhoff I, Bonnet X, Catonne Y, Maestro M, Skalli W. 2009. Skeletal landmarks for tkr implantations: evaluation of their accuracy using eos imaging acquisition system. Orthop Trauma Surg Res. 95 (1):2-11.

Trochu F. 1993. A contouring program based on dual kriging interpolation. Eng Comput. 9:160-177.

Väänänen SP, Grassi L, Flivik G, Jurvelin JS, Isaksson H. 2015. Generation of 3D shape, density, cortical thickness and finite element mesh of proximal femur from a DXA image. Med Image Anal. 24:125-134.

Vatti BR. 1992. A generic solution to polygon clipping. Commun ACM. 7:56-63.

Vincent L. 1998. Minimal path algorithms for the robust detection of linear features in gray images. Ismm. 12:331-338.

Youn K, Park MS, Lee J. 2017. Iterative approach for 3d reconstruction of the femur from un-calibrated $2 \mathrm{~d}$ radiographic images. Med Eng Phys. 50:89-95.

Zheng G. 2010. Statistical shape model-based reconstruction of a scaled, patient-specific surface model of the pelvis from a single standard AP $\mathrm{x}$ ray radiograph. Med Phys. 37:1424-1439.

Zheng G, Hommel H, Akcoltekin A, Thelen B, Stifter J, Peersman G. 2018. A novel technology for $3 \mathrm{~d}$ knee prosthesis planning and treatment evaluation using $2 \mathrm{~d}$ x-ray radiographs: a clinical evaluation. Int J Comput Assist Radiol Surg. 13(8):1151-1158. 